\title{
A METHOD OF MEASURING EARTH RESISTIVITY
}

\author{
By Frank Wenner
}

\section{INTRODUCTION}

A knowledge of earth resistivity ${ }^{1}$ (or specific resistance) may be of value in determining something of the composition of earth, such for example, as moisture content, whether it contains oil, ore of high conductivity, etc., or in the calculation or mitigation of damages to pipe systems by electrolysis caused by the return current of street-railway systems. For some of these or other reasons we may wish to determine the resistivity of limited portions of the earth.

For those cases in which we desire the resistivity of a fairly large portion of earth, extending to a considerable depth, or where there are reasons why we should not disturb the portion of earth to be measured, the following method is suggested: Four holes are made in the earth approximately uniformly spaced in a straight line. The diameter of the holes is not more than ro per cent of the distance between them, and all extend to approximately the same depth, which is usually that at which we are most concerned with the resistivity. In each hole is placed an electrode, which makes electrical contact with the earth only near the bottom, as shown in Fig. I.

This constitutes a four-terminal conductor ${ }^{2}$ the resistance of which depends upon the distance between the electrodes and the

\footnotetext{
1 Here we are concerned with the volume resistivity, which is the resistance of a portion of a conductor having unit length and unit cross section. It is usually expressed as the resistance in ohms of a centimeter cube.

${ }^{2} \mathrm{~A}$ four-terminal conductor is a conductor provided with two terminals to which currect leads may be connected and two terminals to which potential leads may be connected. The resistance of such a conductor is the difference in potential between the potential terminals divided by the current entering and leaving through the current terminals. For a more complete discussion of the four-terminal conductor see this Bulletin, 8, p. 360 , I9r2, Reprint No. IEr.
} 
resistivity, mainly in a region whose linear dimensions are of the same order as the distance between the outside electrodes, but does not depend appreciably upon the size of the electrodes nor the kind of electrical connection they make with the earth.

Therefore, if the depth of the holes, the distance between them, and the resistance (using $I$ and 4 as current and 2 and 3 as potential terminals, or 2 and 3 as current and $I$ and 4 as potential terminals) are measured, we have data from which the effective resistivity in the vicinity can be calculated.

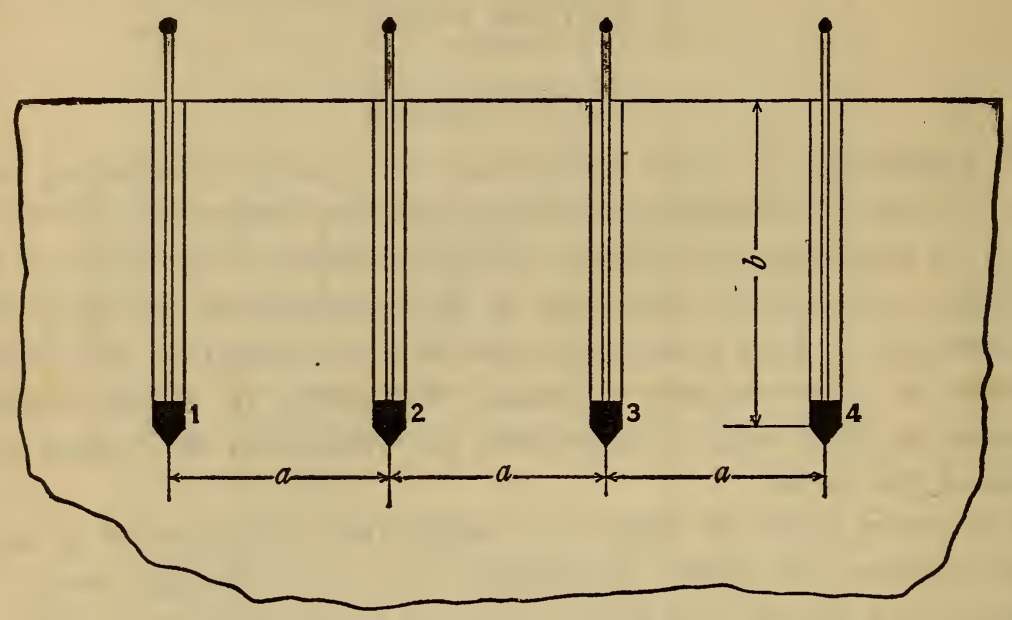

FIG. I.-Diagram showing four electrodes in earth constituting four-terminal conductor as used in measuring earth resistivity

In case $a$ is the distance between. the holes ( 1 to 2,2 to 3 , and 3 to 4 ), $b$ the depth of the holes, $\rho$ the resistivity, and $R$ the measured resistance, then

$$
\rho=\frac{4 \pi a R}{\mathrm{I}+\frac{2 a}{\sqrt{a^{2}+4 b^{2}}}-\frac{2 a}{\sqrt{4 a^{2}+4 b^{2}}}}=\frac{4 \pi a R}{n}
$$

where $n$ has a value between $\mathrm{I}$ and 2 depending upon the ratio of ( $b$ to $a$ ) the depth of the electrodes to the distance between them. Where $b=a, n=1.187 ; b=2 a, n=1.038$; and $b=4 a, n=1.003$.

In case $b$ is large in comparison with $a$,

$$
\rho=4 \pi a R
$$

and in case $b$ is small in comparison with $a$,

$$
\rho=2 \pi a R
$$


If the holes are not in a straight line, or are not of a uniform depth or spacing, the resistivity is easily calculated when the depth of each of the holes and the distances of each from each of the other three are known.

\section{DERIVATION OF EQUATIONS}

To derive equation ( $\mathrm{I}$ ) and the more general relation we may proceed as follows:

Referring to Fig. 2, which is intended to represent a part of an infinite conductor of uniform resistivity, let a unit current enter

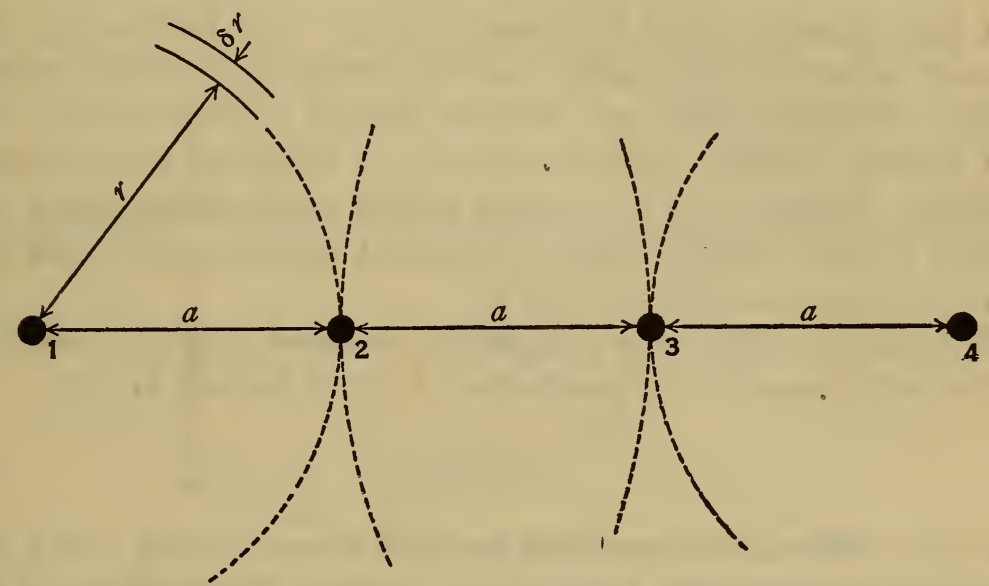

FIG. 2.-Diagram used in showing the relation between the resistivity, resistance, and distances between terminals in an infinite conductor

at the point designated $\mathrm{I}$. This current flows radially away from $I$ and at a distance $r$ its density is $I / 4 \pi r^{2}$. This follows from the fact that at any distance $r$ from I the current density is uniform over the surface of a sphere whose center is at I and whose area is $4 \pi r^{2}$. Since the potential gradient is the current-density times the resistivity,

$$
-\frac{\delta e}{\delta r}=\frac{\rho}{4 \pi r^{2}}
$$

where $e$ is the potential at the distance $r$ from $\mathrm{I}$.

To get the drop in potential, $e^{\prime}-e^{\prime \prime}$ between two points distant $r^{\prime}$ and $r^{\prime \prime}$ from I we must integrate the potential gradient from $r=r^{\prime}$ to $r=r^{\prime \prime}$ or

$$
e^{\prime}-e^{\prime \prime}=\frac{\rho}{4 \pi} \int_{r^{\prime}}^{r^{\prime \prime}} r^{-2} \delta r=\frac{\rho}{4 \pi}\left[\frac{\mathrm{I}}{r^{\prime}}-\frac{\mathrm{I}}{r^{\prime \prime}}\right]
$$


If $e_{x}$ is the drop in potential between the points 2 and 3, distant $a$ and $2 a$ from $\mathrm{I}$, caused by unit current flowing radially from $\mathrm{I}$, equation (5) gives

$$
e_{x}=\frac{\rho}{8 \pi a}
$$

Also if $e_{y}$ represents the drop in potential between 2 and 3 caused by unit current flowing radially toward 4

$$
e_{y}=\frac{\rho}{8 \pi a}
$$

If unit current enters the conductor at I and leaves at 4 , the current density at any point ${ }^{3}$ is the vector sum of the current density resulting from unit current flowing radially from $\mathrm{I}$ and unit current flowing radially toward 4. Likewise, the potential difference between any two points is that which results from unit current flowing radially from I plus that which results from unit current flowing radially toward 4 .

Therefore the difference in potential between 2 and 3 caused by unit current entering the conductor at $\mathrm{r}$ and leaving at 4 is

$$
e_{x}+e_{y}=\frac{\rho}{4 \pi a}
$$

Since the difference in potential for unit current, using I and 4 as current terminals and 2 and 3 as potential terminals, is the resistance $R$,

$$
R=\frac{\rho}{4 \pi a}
$$

$R$ is the resistance of the earth between the equipotential surfaces on which the potential electrodes are placed. It is equal to the resistance of a cylinder of the earth of radius $2 a$ and length $a$.

However, in the measurement of earth resistivity, unless the distance between the electrodes is small in comparison with their distance below the surface, we can not assume that we have an infinite conductor, so equation (9) does not apply.

Referring to Fig. 3, which again represents a part of an infinite conductor, if we let $e_{1}$ be the drop in potential between the points

The current distribution and equipotential surfaces are the same as the "lines of force" and equipo. tential surfaces in the field about two spheres with equal opposite charges. 
2 and 3 , caused by unit current entering the conductor at I, from equation (5) we have

$$
e_{1}=\frac{\rho}{4 \pi}\left[\frac{\mathrm{I}}{r_{12}}-\frac{\mathrm{I}}{r_{13}}\right]
$$

Likewise, if we designate the drop in potential between 2 and 3 , caused by unit current leaving at 4 , by $e_{4}$; that caused by unit

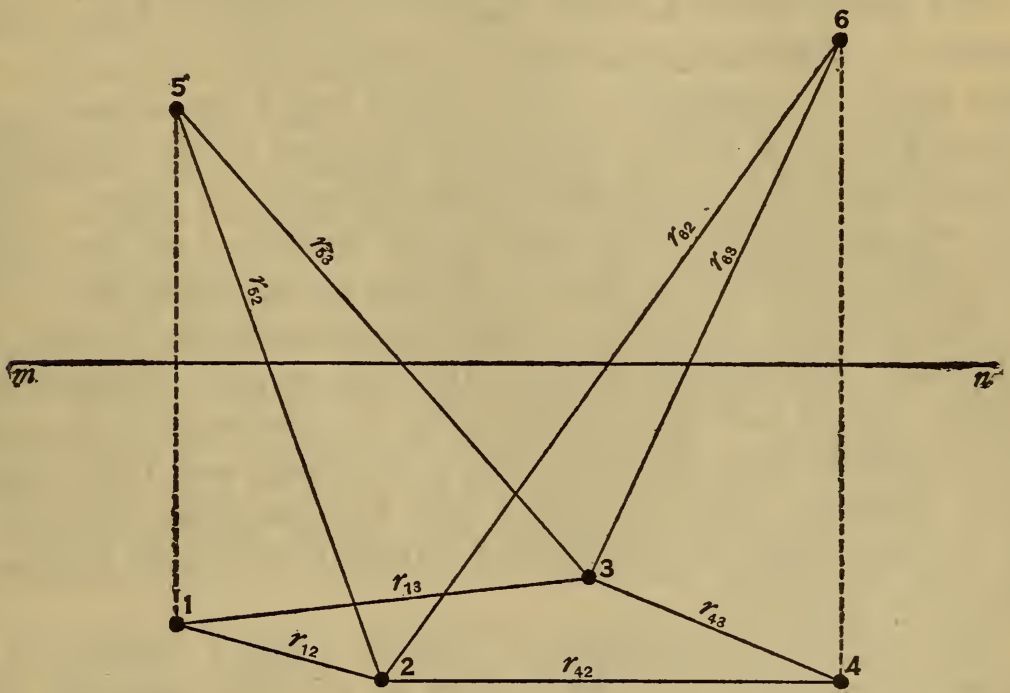

FIG. 3.-Diagram used in showing relation between the resistivity, resistance, and distances between terminals or electrodes in a semiinfinite conductor

current entering at 5 , by $e_{5}$; and that caused by unit current leaving at 6 , by $e_{6}$; we have

$$
\begin{aligned}
& e_{4}=\frac{\rho}{4 \pi}\left[\frac{\mathrm{I}}{r_{43}}-\frac{\mathrm{I}}{r_{42}}\right] \\
& e_{5}=\frac{\rho}{4 \pi}\left[\frac{\mathrm{I}}{r_{52}}-\frac{\mathrm{I}}{r_{53}}\right] \\
& e_{6}=\frac{\rho}{4 \pi}\left[\frac{\mathrm{I}}{r_{63}}-\frac{\mathrm{I}}{r_{62}}\right]
\end{aligned}
$$

Now, if a current $I$ enters at $I$ and leaves at 4 and at the same time an equal current enters at 5 and leaves at 6 , the drop in potential, $E$, between 2 and 3 is $I\left(e_{1}+e_{4}+e_{5}+e_{6}\right)$ or

$$
E=\frac{I \rho}{4 \pi}\left[\frac{\mathrm{I}}{r_{12}}-\frac{\mathrm{I}}{r_{13}}+\frac{\mathrm{I}}{r_{43}}-\frac{\mathrm{I}}{r_{42}}+\frac{\mathrm{I}}{r_{52}}-\frac{\mathrm{I}}{r_{53}}+\frac{\mathrm{I}}{r_{63}}-\frac{\mathrm{I}}{r_{62}}\right]
$$


In the derivation of this equation it has been assumed that I, 2, 3, 4, 5, and 6 represent points. However, the equation can be used without appreciable error where I, 2, 3, 4, 5, and 6 represent metallic electrodes or terminals in a conductor of relatively high resistivity, providing the dimensions of the electrodes are small in comparison with the distances between them.

If 5 and 6 are so located that we can choose a plane (represented by the line $m n$ of Fig. 3) through the conductor, such that the lines connecting $I$ and 5 and 4 and 6 are normal to and bisected by it, it will be evident, on account of the symmetrical arrangement, that no current passes through this plane. Therefore we may remove that part of the conductor on one side of the plane without disturbing conditions on the other side. That is, the equation applies to a semi-infinite conductor having four terminals, providing we consider that the current terminals have images and we take into consideration the distances of the potential terminals both from the current terminals and their images. This, however, does not require that the potential terminals be in the same plane as the current terminals and their images, as shown in the Fig. 3.

Since the drop in potential $E$ between 2 and 3 divided by the current $I$ entering at $I$ and leaving at 4 is the resistance $R$, it follows from equation (I2) that

$$
R=\frac{\rho}{4 \pi}\left[\frac{\mathrm{I}}{r_{12}}-\frac{\mathrm{I}}{r_{13}}+\frac{\mathrm{I}}{r_{43}}-\frac{\mathrm{I}}{r_{42}}+\frac{\mathrm{I}}{r_{52}}-\frac{\mathrm{I}}{r_{53}}+\frac{\mathrm{I}}{r_{63}}-\frac{\mathrm{I}}{r_{62}}\right]
$$

It will, therefore, be evident that the equation gives the relation between the resistivity, resistance, depth, and distance between small electrodes in the earth, as shown in Fig. I, or in the more general case where the electrodes are not in a straight line.

If the electrodes are all at a uniform depth $b$ and at a uniform distance apart $a$ in a straight line, then $r_{12}=a, r_{13}=2 a, r_{43}=a$, $r_{42}=2 a, \quad r_{52}=\sqrt{4 b^{2}+a^{2}}, \quad r_{53}=\sqrt{4 b^{2}+4 a^{2}}, \quad r_{63}=\sqrt{4 b^{2}+a^{2}}$, and $r_{62}=$ $\sqrt{4 b^{2}+4 a^{2}}$. Therefore,

$$
R=\frac{\rho}{4 \pi}\left[\frac{2}{a}-\frac{\mathrm{I}}{a}+\frac{2}{\sqrt{a^{2}+4 b^{2}}}-\frac{2}{\sqrt{4 a^{2}+4 b^{2}}}\right]
$$

which, when solved for $\rho$, gives equation (I). 
In the derivation of these equations we have assumed a uniform resistivity. If the resistivity is not uniform, a solution (except in special cases) is not possible. However, the measured resistance, and consequently the value found for the resistivity, using the equations, depends mainly on the resistivity of the portion of earth between the inner electrodes having a cross section equal to the square of half the distance between the outer electrodes.

\section{MEASUREMENT OF THE RESISTANCE}

Since the electrodes are small, the resistance between the current electrodes and earth, or between any of the electrodes and earth, is large in comparison with the resistance $R$ of the fourterminal conductor, and usually not very constant. Also, since earth acts as an electrolyte there is, in general, an electromotive force in any circuit containing two of the electrodes and polarization at any electrode through which appreciable current passes, even though the current may be alternating. For these reasons none of the usual methods for measuring the resistance of a fourterminal conductor, such as the Thomson bridge and Matthiessen and Hockin bridge methods, seem to meet the conditions very well.

Fortunately there is, in general, no need to measure the resistance to a high accuracy. There is, therefore, no reason why we may not use an ammeter for measuring the current and a voltmeter for measuring the resulting difference in potential between the potential terminals, providing the voltmeter is so used that its readings are not affected by the high resistance between each of these terminals and earth.

The potentiometer arrangement shown in Fig. 4 (using alternating current to obviate the more serious difficulties which might arise on account of polarization with direct current) seems to meet conditions fairly well. The current terminals or electrodes are connected to a source of alternating voltage of suitable value ( 50 to 150 volts for electrodes about 3 or $4 \mathrm{~cm}$ in diameter and 30 to $50 \mathrm{~cm}$ apart). Across the line is connected a transformer stepping down, usually to one-tenth or one-twentieth of the voltage across the current terminals. The low-voltage side of the transformer is connected to the ends of a slide wire, one end 


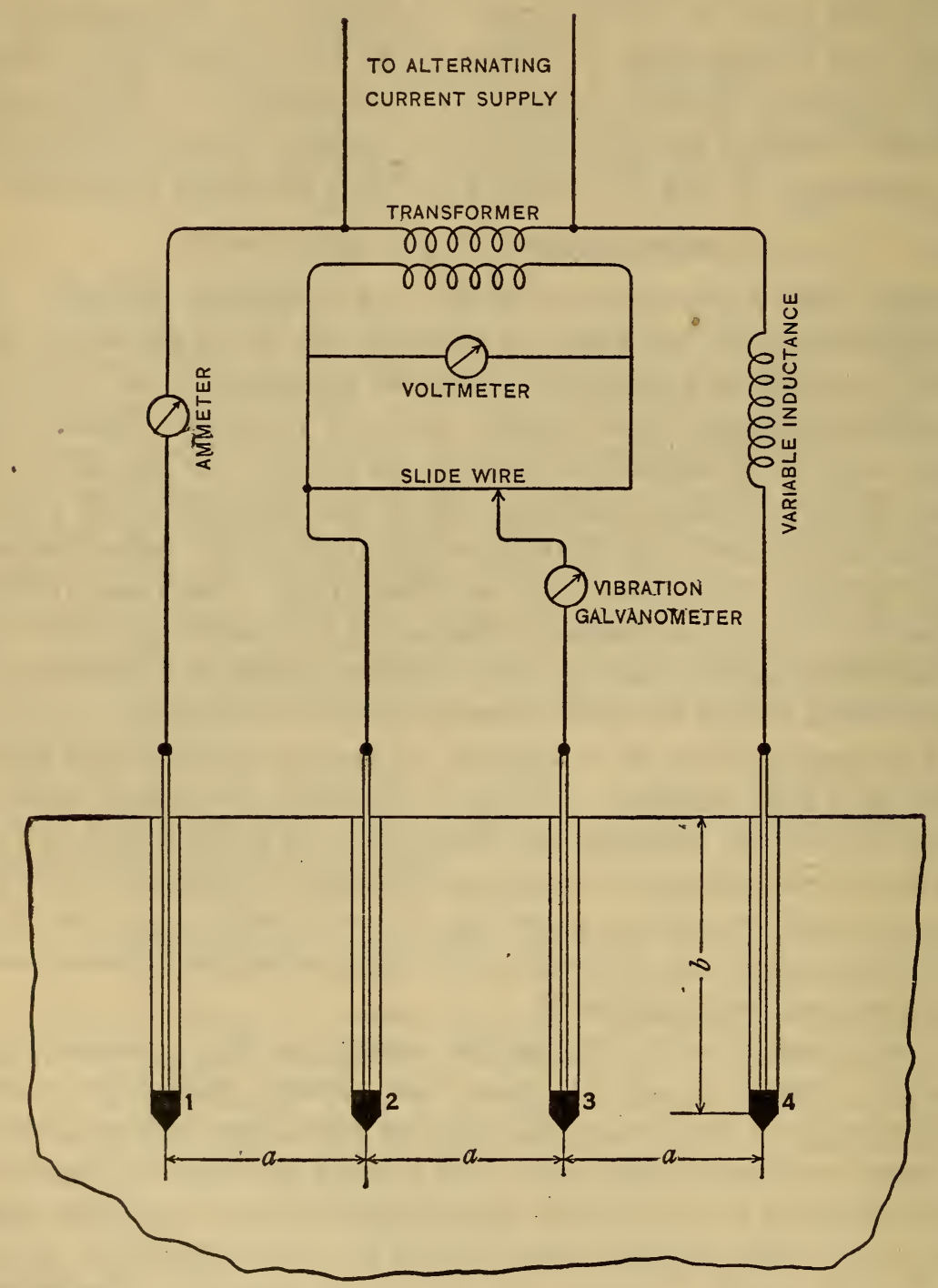

FIG. 4.-Diagram showing arrangement for measuring four-terminal earth resistance by potentiometer-voltmeter-ammeter method 
of which is also connected to one of the potential terminals. The other potential terminal is connected through a vibration galvanometer ${ }^{4}$ (tuned to the frequency of the test current) to the adjustable contact on the slide wire. An ammeter is connected into a lead to one of the current terminals and a voltmeter across the ends of the slide wire, or, if the ratio of the transformer is known when connected to the terminals of the slide wire, the voltmeter may be connected across the line. If the resistance per division of the slide wire is known, we may use an ammeter in series with it instead of the voltmeter.

On account of phase displacement resulting from polarization at the current electrodes, a variable inductance (or inductance with variable resistance in parallel) is connected into one of the leads so that the test current may be brought in phase with the voltage of the low side of the transformer. This is one of the conditions necessary for a zero current through the galvanometer. The other condition is that the magnitude of the $r i$ drop in that part of the slide wire between the adjustable contact and the end connected to one of the potential terminals be equal to the voltage drop across the potential terminals.

If, then, adjustments ${ }^{5}$ are made so that no current flows through the galvanometer, and the position of the sliding contact, the value of the test current, and the voltage across (or current in) the slide wire are read, we have data from which the resistance $R$ is readily calculated.

From the measured resistance and the depth and distance between electrodes the effective resistivity may be calculated as explained above. The result obtained depends mainly upon the resistivity near and between the inner or potential electrodes, and very little upon the resistivity at distances from them equal to or more than the distance between the outer or current electrodes, providing the four electrodes are approximately uniformly spaced.

\footnotetext{
4 If alternating current of from 300 to rooo cycles is available, a telephone receiver could be used to advantage in place of the vibration galvanometer.

${ }^{6}$ As the accuracy required is not high it is not necessary to take special precautions in regard to induced currents, distributed capacity, etc.
} 


\section{USE OF THE METHOD}

The method has been tried out by the author using approximately the arrangement shown in Fig. 4. It has also been used by McCollum and Logan in their investigation of earth resistivity, the results of which, it is expected, will be published soon in one of the Bureau's Technological Papers.

In a particular measurement the electrodes were placed at a depth of approximately $\mathrm{I} 25 \mathrm{~cm}$ and so spaced that (referring to Fig. 3) $r_{12}=25, r_{13}=58, r_{43}=3 \mathrm{I}$, and $r_{42}=64 \mathrm{~cm}$. This gives $25 \mathrm{I}$ $\mathrm{cm}$ for $r_{52}$ and $r_{63}$, and $255 \mathrm{~cm}$ for $r_{53}$ and $r_{62}$. These values substituted in equation (I4) give

$$
R=\frac{\rho}{4 \pi} .0397 \text { or } \rho=316 R
$$

The measured value of $R$, the mean of several ratios of potential difference to current, was found to be 25.3 , which give 8000 for $\rho$, the resistivity expressed as the mean resistanice in ohms of a centimeter cube.

So far the method has been used only for determining resistivities in a region a few meters or less in radius and very close to the surface. To measure the effective resistance of a much larger portion of earth, extending to a considerable depth, the electrodes would be placed much farther apart. By using large electrodes a considerable distance apart, a large test current and a very sensitive detector (either of the vibration galvanometer or the separately excited dynamometer type) it should be possible to get some idea of the effective resistivity from the surface to a considerable depth, even though the electrodes are placed practically on the surface. The result obtained would depend to a considerable extent upon the resistivity of a surface layer should it be low, as is usually the case. Tre effect of the surface layer could be estimated roughly from the results obtained from measurements with the electrodes closer together, and thus some idea obtained as to the resistivity at different depths. Such a measurement might be of assistance in locating deposits of ore of high conductivity.

WASHINGTON, JULY I 5 , I9I 5 . 